\title{
Effect of Shear Deformation on Microstructural Evolution of Ni-30Fe Alloy during Hot Deformation
}

\author{
Jae-Young Cho ${ }^{1,2}$, Tadanobu Inoue ${ }^{1}$, Fuxing Yin $^{1}$ and Kotobu Nagai ${ }^{1}$ \\ ${ }^{1}$ Steel Research Center, National Institute for Materials Science, Tsukuba 305-0047, Japan \\ ${ }^{2}$ School of Materials Science and Engineering, Seoul National University Shinrim-dong, \\ San 56-1, Kwanak-ku, Seoul 151-742, Korea
}

Electron backscattered diffraction analysis has been used to investigate the effect of shear deformation on the microstructural evolution of a Ni-30Fe alloy during hot deformation. The alloy was compressed by $50 \%$ or $75 \%$ in thickness at a strain rate of $1 / \mathrm{s}$ in a single pass at $1023 \mathrm{~K}$ using a hot compression simulator. An explicit finite element analysis was carried out to evaluate the inhomogeneous strain distribution introduced in the specimens by hot compression simulator. As the equivalent strain increased, the fraction of high angle grain boundaries with misorientaions between $15^{\circ}$ and $30^{\circ}$ increased almost in the similar way regardless of the presence of shear strain. The fraction of high angle grain boundaries having misorientations in excess of $30^{\circ}$ increased mainly at the expense of low angle grain boundaries with misorientations smaller than $15^{\circ}$. Such the expense occurred at much higher rate with shear strain than without shear strain. The compressive direction changed continuously in the areas with shear strain component during deformation, which was thought to accelerate the subdivision of austenite grain interiors with increased misorientations between subdivided local areas.

(Received April 6, 2004; Accepted June 23, 2004)

Keywords: nickel-30 ferrous alloy, microstructural evolution, high angle grain boundary, low angle grain boundary, electron back-scattering diffraction pattern

\section{Introduction}

In recent years, several severe deformation techniques have been proposed, for example, equal channel angular extrusion (ECAE), ${ }^{1-3)}$ multi-axis compression, ${ }^{4)}$ accumulative roll bonding $(\mathrm{ARB})^{5-7)}$ and severe plastic torsion straining (SPTS) ${ }^{8)}$ These techniques aim to accumulate high plastic strains into materials since the increase of the plastic strains leads to the enhanced rate of grain refinement.

The plastic strain is generally composed of two strain components or has two deformation modes: shear strain and the compressive (or tensile) strain. It is expected that these two strain components coexist in the materials deformed by the severe deformation techniques. The shear strain is known to be more effective for creating finer ferrite grains than the compressive strain due to the faster increasing rate of austenite grain boundary surface area per unit volume. ${ }^{9)}$ Recently, Inoue et al. have reported that under the same equivalent plastic strain $\varepsilon_{e q}$, the ferrite grain size in the area with the shear strain is finer than that in the area without the shear strain. ${ }^{10,11)}$ At the same time it is also expected that the different type of plastic strain will introduce significant changes in the microstructural evolution behavior of austenite grain interiors.

In this study, the effect of deformation mode on the microstructural evolution of $\mathrm{Ni}-30 \mathrm{Fe}$ alloy hot compressed by a pair of anvils is investigated. The explicit finite element analysis was used to evaluate the strain distribution in the specimens compressed by reduction ratios of $50 \%$ and $75 \%$. On the basis of the calculated strain distribution, the microstructural evolution behaviors in the positions with and without the shear strain were compared according to the equivalent plastic strain $\varepsilon_{e q}$. Finally, the effect of the shear strain on the microstructural evolution was investigated in the range of $0=\gamma_{x y}=2.29$ at the fixed equivalent strain $\varepsilon_{e q}$ of 1.5 by means of EBSD technique.
Table 1 Chemical compositions of specimen (in mass\%).

\begin{tabular}{ccccccc}
\hline $\mathrm{C}$ & $\mathrm{Mn}$ & $\mathrm{Si}$ & $\mathrm{P}$ & $\mathrm{S}$ & $\mathrm{Ni}$ & $\mathrm{Fe}$ \\
\hline 0.002 & $<0.002$ & 0.008 & 0.002 & 0.001 & 69.8 & Bal. \\
\hline
\end{tabular}

\section{Experimental}

\subsection{Specimen preparation}

The ingot of Ni-30Fe alloy was prepared in a vacuum induction melting furnace with a weight capacity of $50 \mathrm{~kg}$. The chemical compositions of Ni-30Fe alloy are given in Table 1 . The ingot was re-heated to $1473 \mathrm{~K}$ and hot rolled to make a plate with a size of $T 60 \times L 550 \times W 140(\mathrm{~mm})$. The final rolling temperature was $1243 \mathrm{~K}$. Rectangular specimens that were $12 \mathrm{~mm}$ in thickness, $20 \mathrm{~mm}$ in width and $18 \mathrm{~mm}$ in length were machined from the hot rolled plate for hot compression test.

\subsection{Hot compression test}

Figure 1 shows the path of the hot compression test. A hot compression simulator (DSI, Gleeble 2000) was used for the thermo-mechanical treatment. The specimen was heated to $1023 \mathrm{~K}$ and held for 20 seconds to make the specimen temperature uniform and then deformed by reduction ratios of $50 \%$ and $75 \%$ at a strain rate of $1 / \mathrm{s}$. The deformation temperature of $1023 \mathrm{~K}$ was known to be in non-recrystallized region. ${ }^{12)}$ The deformed specimen was quenched to room temperature by water jet immediately after the compression to freeze the deformed microstructure.

\subsection{Observation of microstructural characteristics}

The specimen surface observed was parallel to the compression direction, as shown in Fig. 2. The specimens were etched for 20 seconds using a solution containing $\mathrm{HCl}$ 


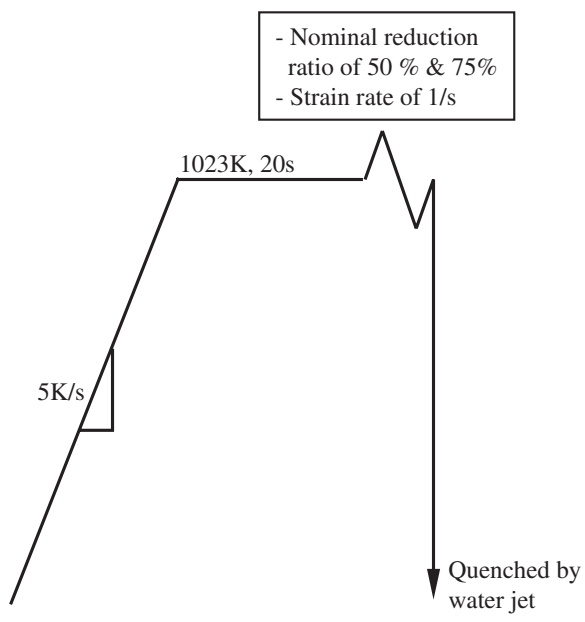

Fig. 1 Hot compression process.

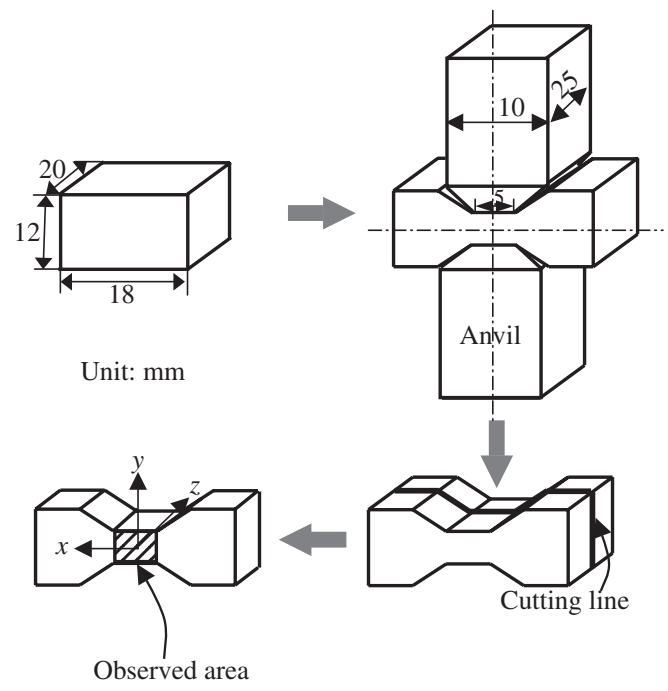

Fig. 2 Schematic drawing of compression test and observed area of deformed specimen.

(10 $\mathrm{ml}), \mathrm{FeCl}_{3}(5 \mathrm{~g})$ and ethanol $(40 \mathrm{ml})$. The microstructures were characterized by optical microscopy and scanning electron microscopy.

The grain orientations were determined by scanning electron microscopy (LEO Gemini 1550) using the TSL EBSD system. The specimens for the EBSD examination were ground with $\mathrm{SiC}$ papers, mechanically polished with a $1 \mu \mathrm{m}$-diameter alumina paste, and then electrochemically polished for $20 \mathrm{~s}$ with a solution of $10 \%$ perchloric acid in ethanol. The maps were typically $150 \mu \mathrm{m} \times 150 \mu \mathrm{m}$ with a step size of a $0.5 \mu \mathrm{m}$. Because the angular accuracy of the present EBSD system is limited to approximately $1.5^{\circ}$ in the expression of misorientation of two neighbor grains, in all the maps the following definitions were used. Low angle grain boundaries (LAGBs), or subgrain boundaries, were defined as boundaries with misorientation between $1.5^{\circ}$ and $15^{\circ}$ misorientation, and high angle grain boundaries (HAGBs), or grain boundaries, are defined as boundaries greater than $15^{\circ}$ in the misorientation.

\section{Numerical Analysis}

The three-dimensional dynamic finite element simulation was carried out using the explicit FE code ABAQUS/ Explicit. ${ }^{13)}$ The specimen is assumed to be isotropic and homogeneous. The anvil is regarded as a rigid body and the specimen is not constrained in the $x$ direction. An 8 node linear element was used for the specimen. And the finite element mesh in the sample includes 6552 nodes and 5400 elements. Furthermore, adaptive meshing is carried out in the analysis because the mesh becomes too degenerate on heavy deformation.

The stress-strain relationships employed in the analysis were measured experimentally by compression test using a cylindrical specimen, and modified by considering barreling. ${ }^{10)}$ In the analysis, the frictional conditions on the contacting planes between the anvil and the specimen are described by the Coulomb model. The friction coefficient was assumed to be constant throughout the deformation and was determined by using experimental results with a screw. The Coulomb condition with a friction coefficient of 0.3 is used as the frictional condition on the contacting planes between the anvil and the specimen. Refs. 10-11) may be consulted for more detailed description of the numerical analysis.

\section{Results and Discussion}

\subsection{Plastic strain distribution estimated by numerical analysis}

Figure 3 represents the contour maps showing the distributions of shear strain $\gamma_{x y}$ and equivalent plastic strain $\varepsilon_{e q}$ after $50 \%$ and $75 \%$ deformations at $1023 \mathrm{~K}$ and a strain rate of $1 / \mathrm{s}$. Here, the $\gamma_{x y}$ is denoted as the engineering strain. The numerical analysis was carried out on the $x-y$ plane of the gray quarter part of the specimen. As can be seen in Fig. 3, the material expands in the $x$ direction and contracts in the $y$ direction by hot compression test. The shear strain increases towards the anvil edge from the center. And the shear strain becomes zero along the $x$ and $y$ axes in both cases of $50 \%$ and $75 \%$ deformations, which means that the plastic strains along the $x$ and $y$ direction show a plane strain condition, i.e., $\varepsilon_{x x}+\varepsilon_{y y}=0, \varepsilon_{z z}=0$. The equivalent plastic strain shows the maximum value at the specimen center and the anvil edges. The high compressive strains at the center and the high shear strains at the anvil edges seem to be responsible for the maximum equivalent plastic strain.

Microstructures at different sites on the cross-section surface of the $75 \%$ compressed specimen are shown in Fig. 4. The microstructure below the contacting surface with anvils shows non-deformed austenite grains of about $90 \mu \mathrm{m}$ in diameter. However, the specimen is heavily deformed around the central region and anvil edges, as expected in Fig. 3 and the deformation feature shows a symmetrical distribution about the $x$ and $y$ axis. Figures 4(b)-(d) show the magnified optical micrographs. They show different deformed microstructures from site to site. The difference in the deformed microstructures is due to the strain variation induced by hot compression, as seen in Fig. 3.

Figure 5 represents schematic diagrams showing the sites 


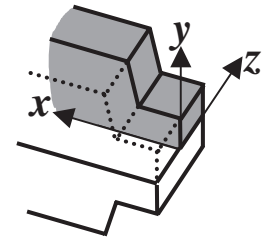

(a)

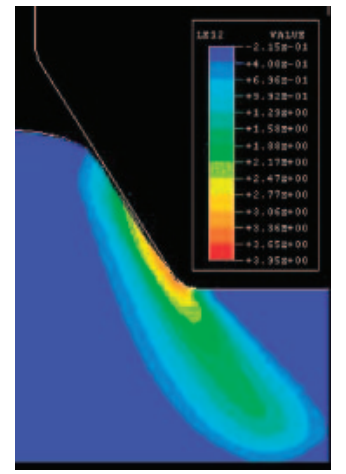

(b)

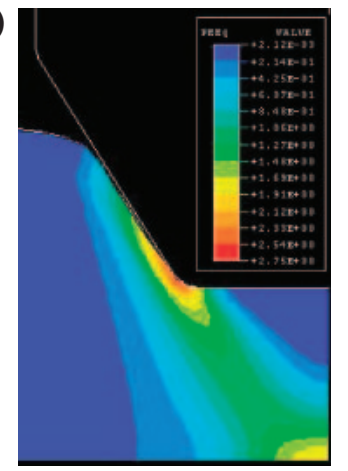

(c)

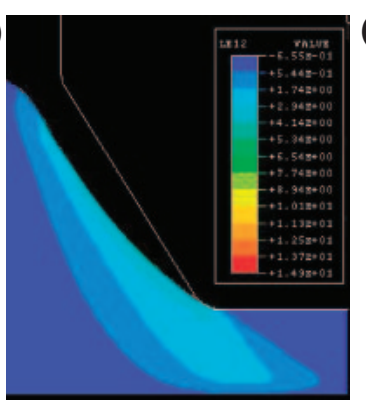

(d)

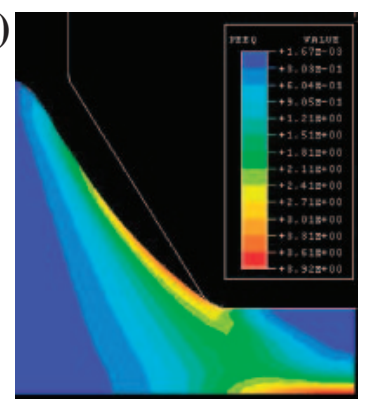

Fig. 3 Contour maps of (a) shear strain $\gamma_{x y}$ and (b) equivalent plastic strain $\varepsilon_{e q}$ after $50 \%$ compression and (c) shear strain $\gamma_{x y}$ and (d) equivalent plastic strain $\varepsilon_{e q}$ after $75 \%$ compression at $1023 \mathrm{~K}$ and strain rate of $1 / \mathrm{s}$.

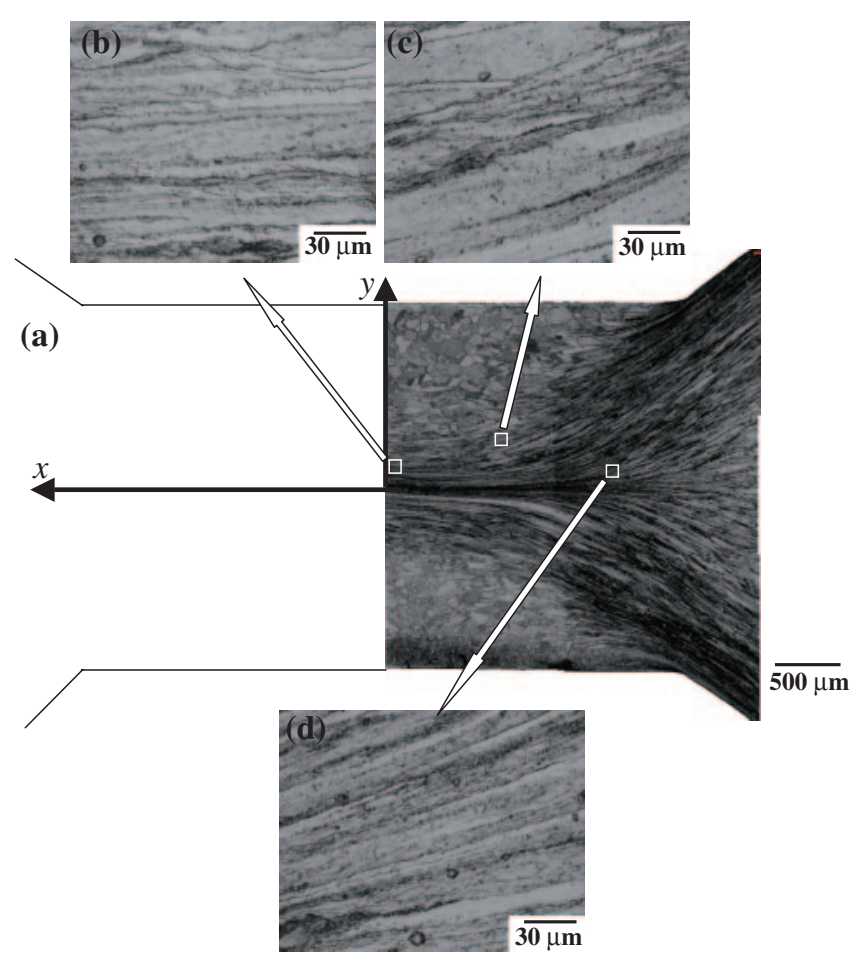

Fig. 4 (a) Optical micrograph after deformation of $75 \%$ at $1023 \mathrm{~K}$ and strain rate of $1 / \mathrm{s}$ and (b)-(d) magnified views of areas indicated by squares in (a).

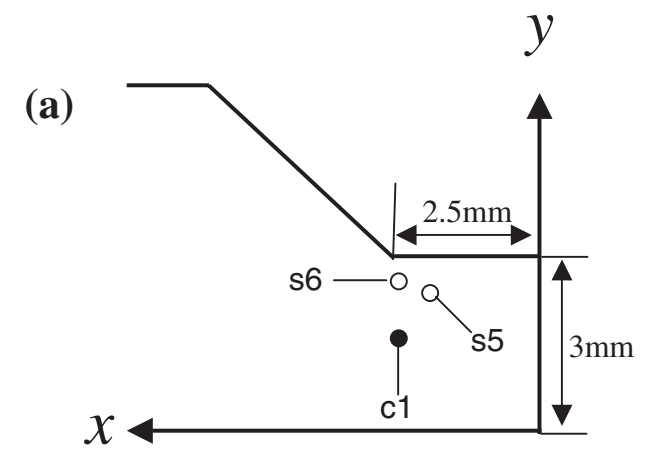

(b)

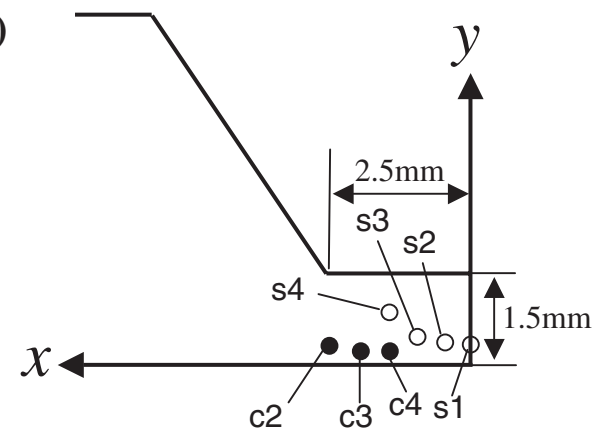

Fig. 5 Schematic diagrams of cross sections of specimens after (a) $50 \%$ and (b) $75 \%$ compression.
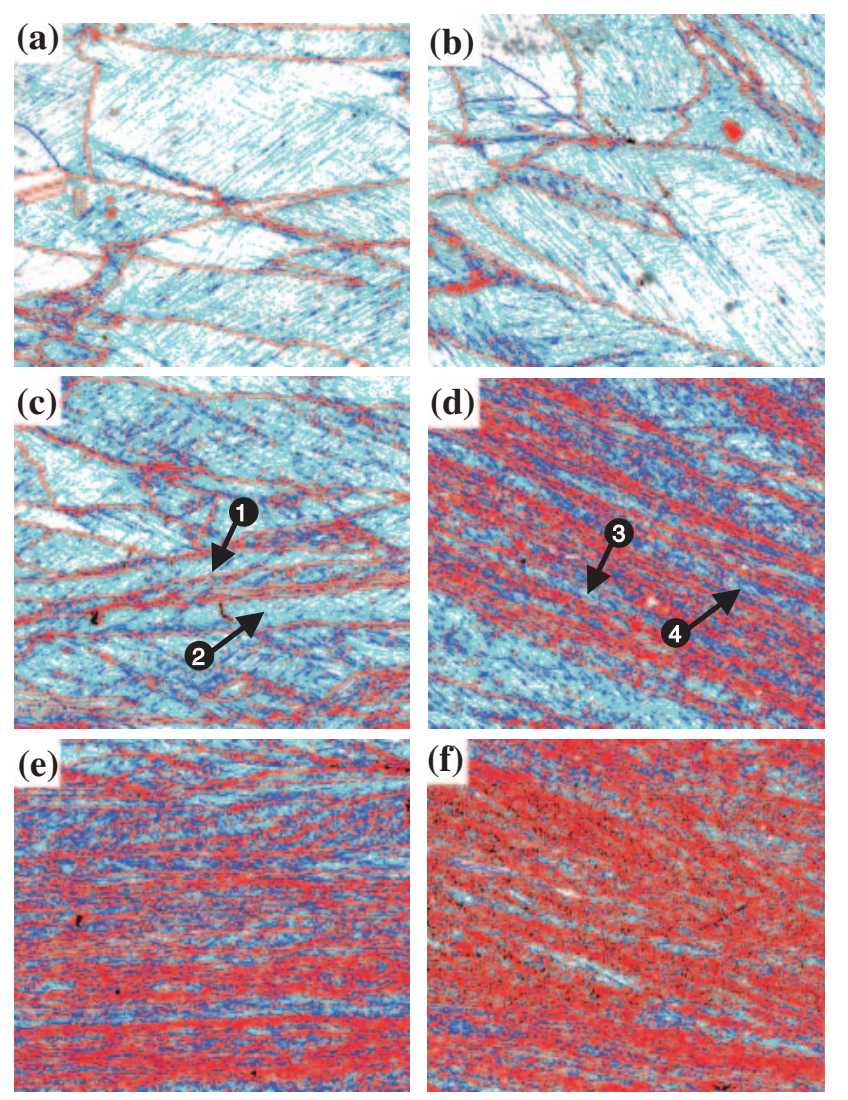

$\left(1.5^{\circ} \leq \theta<5^{\circ}\right)$

$\left(5^{\circ} \leq \theta<15^{\circ}\right)$

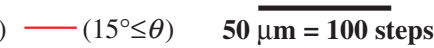

Fig. 6 EBSD maps showing the development of deformation structures according to equivalent strains (a), (b) $\varepsilon_{e q}=0.68$ (c), (d) $\varepsilon_{e q}=1.70$ and (e), (f) $\varepsilon_{e q}=2.83$; in (a), (c) and (e) no shear strain; in (b), (d) and (f) shear strain $\gamma_{x y}$ of 1.0. HAGBs are shown as red lines, and LAGBs as green lines. 
Table 2 Calculated shear strain $\gamma_{x y}$, compressive strain $\varepsilon_{y y}$ and equivalent strain $\varepsilon_{e q}$ of the sites indicated in Fig. 5.

\begin{tabular}{cl}
\hline Position & \multicolumn{1}{c}{ Calculated strains } \\
\hline $\mathrm{C} 1$ & $\varepsilon_{e q}=0.68, \varepsilon_{y y}=-0.33$ \\
\hline $\mathrm{C} 2$ & $\varepsilon_{e q}=1.70, \varepsilon_{y y}=-1.37$
\end{tabular}$\quad \gamma_{x y}=1.0$

where the compressive strains and the shear strains and the equivalent plastic strains are evaluated in detail. The calculated strains at each site are shown in Table 2. The solid symbols in Fig. 5 indicate the positions in which the equivalent strain varies under the fixed shear strain of 1.0. On the other hand, the open symbols represent the positions where the shear strain varies under the same equivalent plastic strain of 1.5 .

The following sections will deal with the microstructural evolution behaviors in the positions with and without the shear strain and the effect of the shear strain on the microstructural evolution in the range of $0=\gamma_{x y}=2.29$ at the fixed equivalent strain $\varepsilon_{e q}$ of 1.5 .

\subsection{Evolution of deformed structure with strain}

Figure 6 presents the EBSD maps of the deformed specimens by three equivalent plastic without or with shear strain. The left hand side corresponds to the area along the $y$ axis of Fig. 4(a), showing a plane strain compression without shear strain. On the other hand, the right hand side corresponds to the sites of $\mathrm{C} 1-\mathrm{C} 4$ of Fig. 5 with shear strain $\gamma_{x y}$ of 1.0. It can be seen that at an equivalent plastic strain $\varepsilon_{e q}$ of 0.68 (Figs. 6(a), (b)), both deformed structures within austenite grains are composed of only LAGBs. At an equivalent plastic strain of 1.70 (Fig. 6(c), (d)), the laminar elongation of the structure aligned in the material flow direction becomes more evident. New HAGBs were generated by the formation of deformation bands within austenite grains, signifying localized non-homogeneous deformation. However, the considerably elongated austenite grains of \#1-\#4 indicated by arrows in Figs. 6(c) and (d), have no HAGBs in contrast to other austenite grains. These elongated austenite grains with uniform deformation structure are thought to develop in the austenite grains with stable crystal orientation. But such the stable elongated grains are known to be broken into subgrains when they are reduced to approximately one subgrain size in width. ${ }^{14)}$ With increasing the equivalent plastic strain to 2.83 (Figs. 6(e), (f)), the grains are subdivided more rapidly by the formation of HAGBs in almost all the austenite grains.

In order to investigate the effect of shear strain component on the microstructural evolution, EBSD maps was determined for the sites of S1-S6 of Fig. 5 with shear strain $\gamma_{x y}$ up to 2.29, under the same equivalent strain $\varepsilon_{e q}$ of 1.5 as shown in Fig. 7. With fixing the equivalent plastic strain at 1.5, the amount of compressive strain decreases with increasing shear strain, as shown in Table 2. Since it becomes difficult to discern the austenite grain boundaries with increasing strain, SEM micrographs are also shown in Fig. 7. It can be seen that the site S1 with a shear strain $\gamma_{x y}$ of 0 , that is, deformed with just compressive strain, new HAGBs were generated by the formation of deformation bands within austenite grains, as indicated by arrows. After deformation with shear strain of 1.92 (at site S5), some grains, indicated by arrows, were filled with fine HAGBs. With increasing the shear strain to 2.29 (at site S6), new HAGBs get developing on a much finer scale within almost all the austenite grains.

From Fig. 7, it can be also known that the compressive direction varies with shear strain, contrary to no rotation of compressive direction in the sites without it. In order to express local strain, it is convenient to introduce a local coordinate system (1-2-3) whose axes coincide with the directions of the principal strains $\varepsilon_{1}, \varepsilon_{2}$ and $\varepsilon_{3}\left(\varepsilon_{1}<\varepsilon_{2}<\varepsilon_{3}\right)$. Using each strain value obtained in the specimen coordinate system $(x-y-z)$, the directions of the principal axes in the local coordinate system and the principal strains are calculated. The schematic drawings of the principal axes (1-3) and the rotation angle $\phi$ from the $x$ and $y$ axes to the principal axes at each site are also illustrated in Fig. 7.

Under the same equivalent strain of 1.5 , the principal strains at each site are almost identical around 1.2 with different shear strains from 0 to 2.29. Accordingly the deformed austenite grains have a similar thickness of approximately $25 \mu \mathrm{m}$ as indicated by arrows in SEM micrographs of Fig. 7.

On the other hand, the rotation angle $\phi$ calculated with shear strain becomes larger with increasing shear strain. For example, the rotation angle is $10^{\circ}$ at the shear strain of 1.08 and $46^{\circ}$ at the shear strain of 2.29. And further it shows a good agreement with the slope of the deformed austenite grain boundary when the SEM micrograph and the illustration of rotation angle in Fig. 7 are compared.

These results imply that the compressive direction rotates continuously during deformation and a kind of multidirectional deformation occurs with an increase in shear strain. Multidirectional deformation is well known to be effective for grain refinement. ${ }^{18)}$ That is, the multidirectional deformation is believed to induce multiplication of slip systems and enhance the formation of high angle grain boundaries or the subdivision of grains. Consequently, even under the same equivalent strain, the increasing the shear strain might accelerate the subdivision of austenite grain interiors with increased misorientations between subdivided local areas.

\subsection{Statistical measurements of the microstructural evolution during deformation}

The fractions of HAGB area are displayed as a function of equivalent plastic strain in Fig. 8. Figure 8 shows that the fraction of HAGB increases as the equivalent plastic strain increases. And the existence of shear strain makes the higher increase rate of increase in HAGB area fraction than no shear strain. At the equivalent plastic strain of 2.83, the fraction of HAGB is over $50 \%$ with shear strain of 1.0 , in comparison 


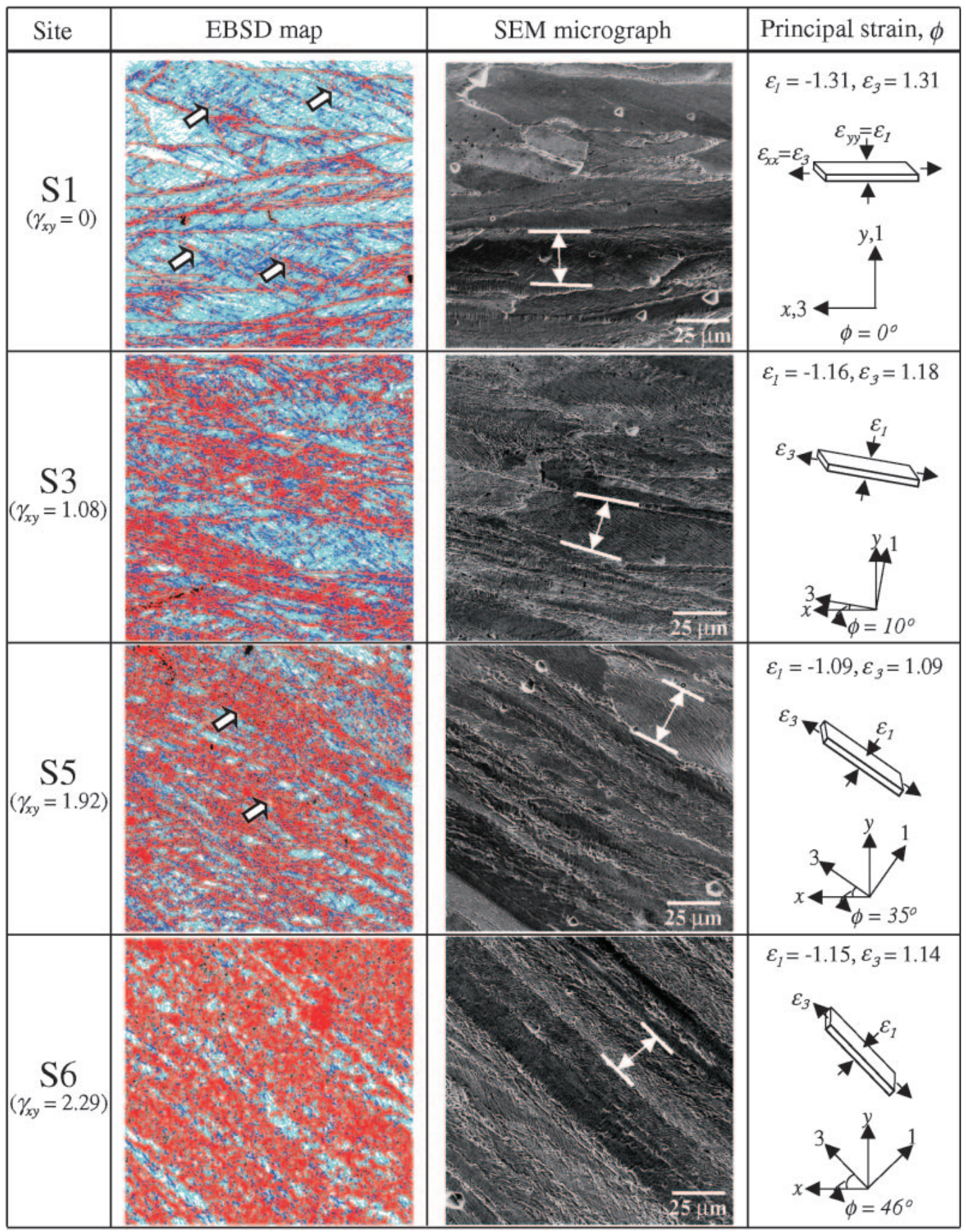

Fig. 7 EBSD maps, SEM micrographs and principal strains for some sites at equivalent strain of $\varepsilon_{e q}=1.5$ : site S1 $\left(\gamma_{x y}=0\right)$, site S3 $\left(\gamma_{x y}=1.08\right)$, site S5 $\left(\gamma_{x y}=1.92\right)$ and site S6 $\left(\gamma_{x y}=2.29\right)$ and $\phi$ means the rotation angle from $x, y$ axes to principal axes.

with only $30 \%$ without the shear strain. Even with the increased equivalent plastic strain of 3.5, the fraction of HAGB is still $43 \%$ HAGB without the shear strain.

The typical boundary misorientation distribution determined from the EBSD maps is shown in Fig. 9 at an equivalent plastic strain of 2.83 with and without shear strain. The graphs show the relative frequency of boundary misorientations at intervals of $3^{\circ}$. Misorientations less than $1.5^{\circ}$ are omitted due to the orientation noise in EBSD measurements. The fraction of misorientation angle, especially between $30^{\circ}$ and $50^{\circ}$, is approximately two times with shear strain as large as without shear strain.
Figure 10 compares the rates of development of boundaries for each misorientation range with increasing equivalent plastic strain in the area with and without shear strain component during deformation. In the graphs two classes of HAGBs are shown; lower high angle grain boundaries with misorientations between $15^{\circ}$ and $30^{\circ}$, and very high angle grain boundaries with misorientations in excess of $30^{\circ}$. The LAGBs are plotted against the left vertical axis and all the HAGBs are plotted against the right in Fig. 10.

As the equivalent strain increases, the fraction of HAGB $\left(15^{\circ}-30^{\circ}\right)$ increases almost in the similar way regardless of the presence of shear strain. The fraction of HAGB $\left(>30^{\circ}\right)$ 


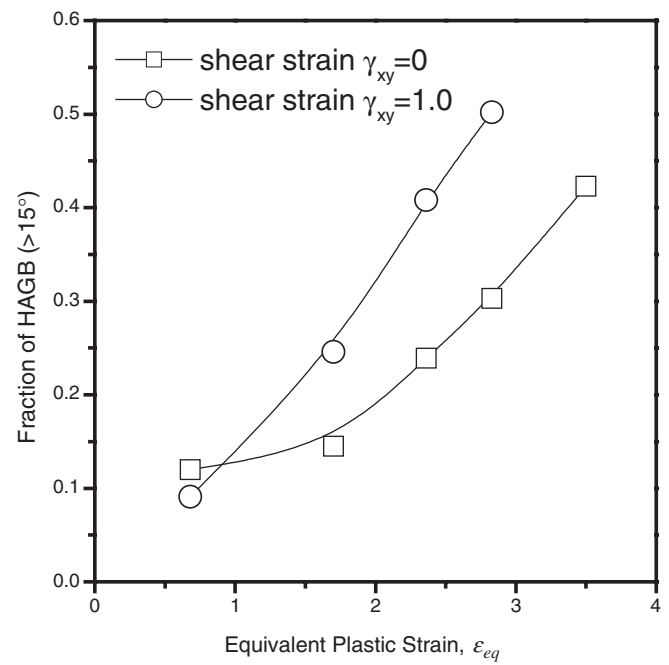

Fig. 8 Fraction of HAGB area plotted as a function of equivalent plastic strain with and without shear strain.

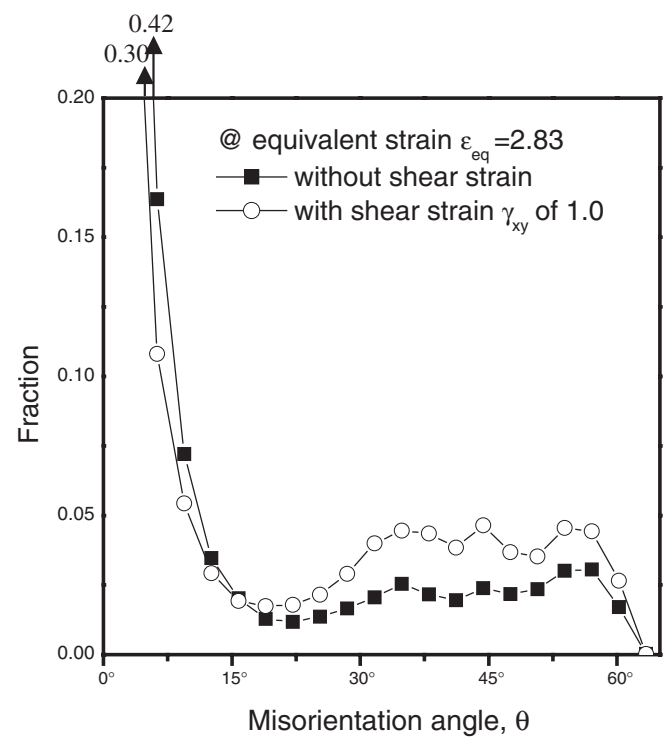

Fig. 9 One example of boundary misorientation distributions at equivalent plastic strain of 2.83 with and without shear strain of 1.0 .

increases mainly at the expense of LAGB $\left(<15^{\circ}\right)$. Such the expense occurs at much higher rate with shear strain than without shear strain.

Examples of misorientation distributions are presented in Fig. 11 for three shear strain levels at an equivalent plastic strain of 1.5. The fraction of LAGB decreases with an increase in shear strain. For example, the fraction of LAGB is $69 \%$ without shear strain but $33 \%$ with the shear strain of 2.29. On the other hand, the fraction of HAGB $\left(>30^{\circ}\right)$ remarkably increases with an increase in shear strain.

Figure 12 represents the change of the fraction of grain boundary misorientation by shear stain when the equivalent strain is 1.5. Again the fraction of HAGB $\left(>30^{\circ}\right)$ increases mainly at the expense of $\operatorname{LAGB}\left(<15^{\circ}\right)$ with an increase in shear strain.

In general, the increase in the HAGBs during deformation is attributed to two mechanisms; (i) the formation of new (a)

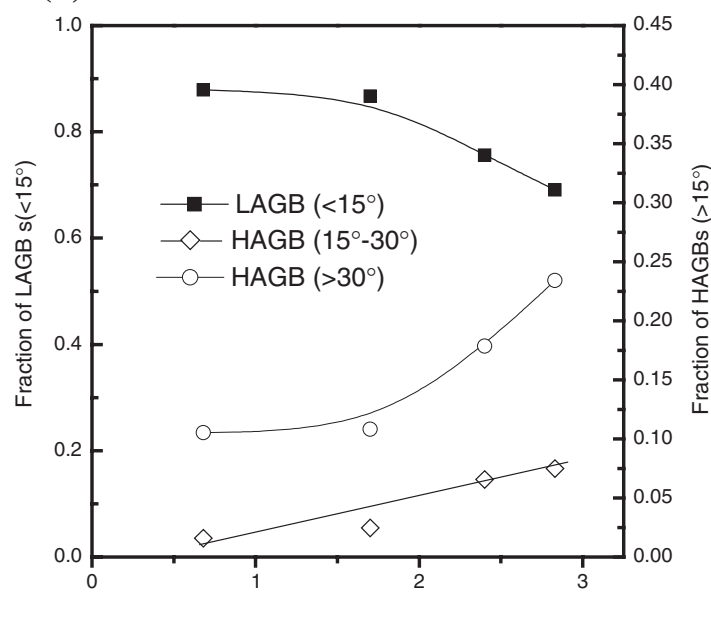

Equivalent Plastic Strain, $\varepsilon_{e q}$

(b)

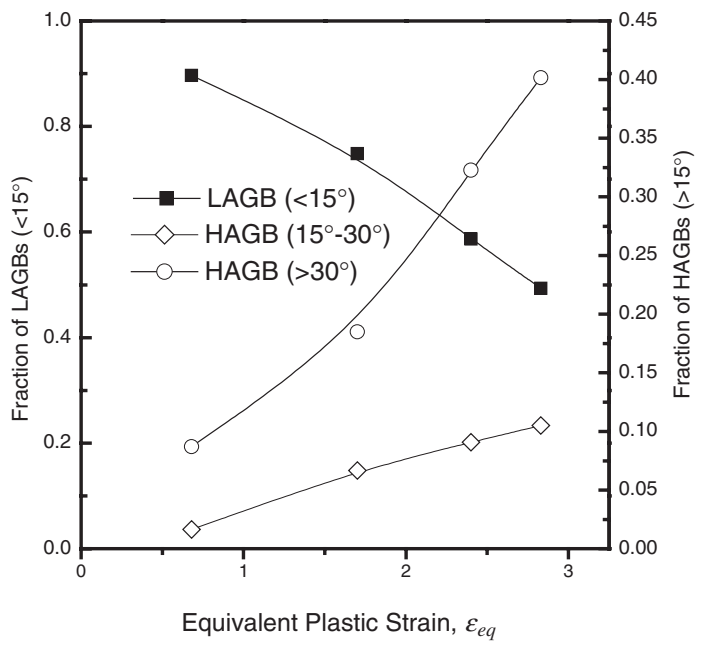

Fig. 10 Comparison of the rates of development of boundary area fractions according to equivalent strains (a) without and (b) with shear strain of 1.0.

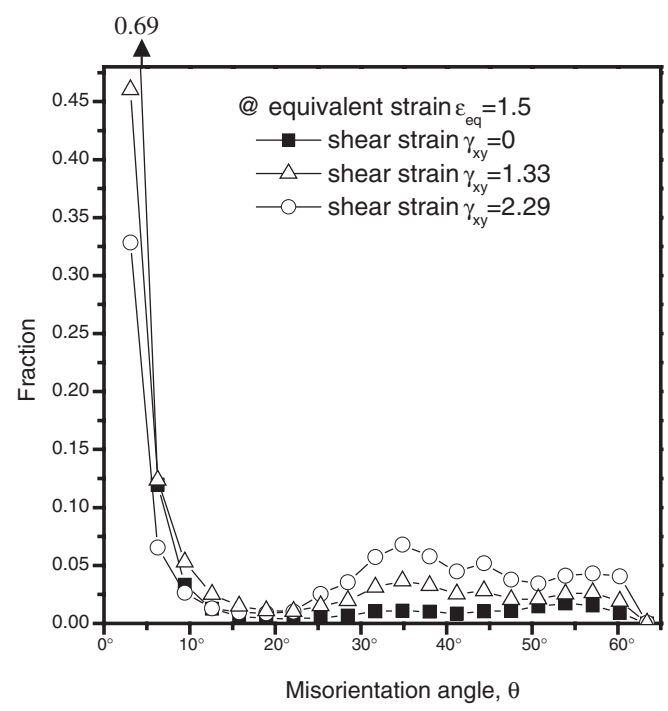

Fig. 11 Misorientation distributions according to shear strains at equivalent plastic strain of 1.5 . 


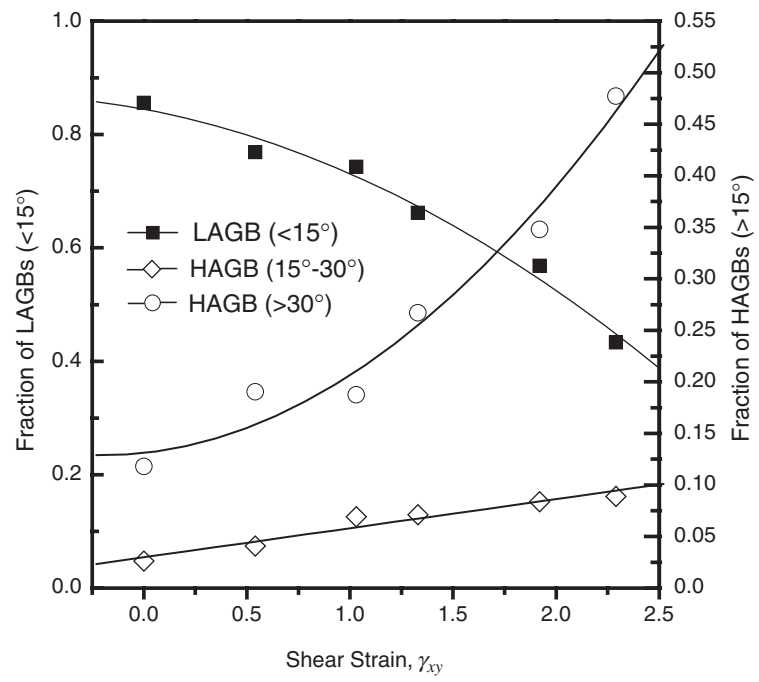

Fig. 12 Rates of development of boundary area fractions according to shear strains at equivalent plastic strain of 1.5 .

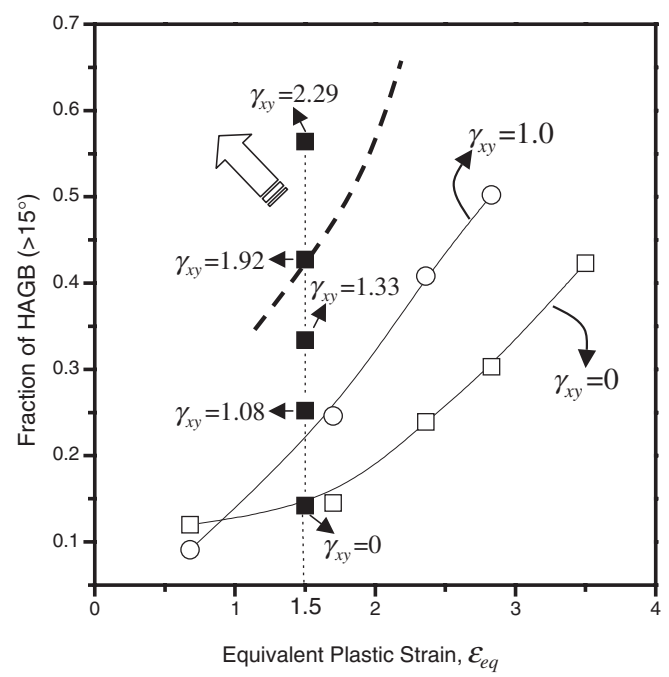

Fig. 13 Effect of shear strain on fraction of HAGB area, plotted as a function of equivalent plastic strain.

high angle grain boundaries by grain subdivision ${ }^{9-11)}$ and (ii) the extension of the initial and newly formed grain boundaries with increasing strain. ${ }^{15-20)}$ Here the initial grain size is known to be a significant factor. ${ }^{16)}$ Namely, the former process is expected to occur predominantly in materials with large grain sizes (i.e. $>25 \mu \mathrm{m}$ ). Because the grain size of interest in the present study was approximately $90 \mu \mathrm{m}$, the former is thought to be controlling in the formation of HAGBs area of Figs. 6 and 10, as confirmed by SEM micrographs of Fig. 7. The formation of new HAGBs by grain subdivision has been widely studied. ${ }^{15-20)}$ Initially at low strains very coarse deformation bands are formed. As the strain increases cell blocks bordered by dense dislocation walls develop within the coarse bands and rotate progressively. On average the misorientation between adjacent cell blocks increases with strain and some dense dislocation walls will evolve into new high angle boundaries.
Figure 13 represents the fractions of HAGB area according to shear strain, with fixing the equivalent plastic strain at $\varepsilon_{e q}=1.5$, together with the data of Fig. 8 which showed the faster formation of high angle grain boundaries during deformation due to the presence of shear strain of $\gamma_{x y}=1.0$. It is likely that the curve passing through the point indicated by $\gamma_{x y}=1.92$ might follow the dotted bold line with increasing equivalent plastic strain. And the curves will move in the upper left-handed direction with an increase of shear strain component, as indicated by the arrow in Fig. 13. Hence, it is implied that the same HAGB fraction can be reached much rapidly at a relatively small equivalent plastic strain, with increasing the shear strain component.

The present study clarified that the shear strain component is more effective to develop uniform HAGBs in austenite grain interiors than the compressive strain.

\section{Conclusions}

On the basis of the calculated strain distribution induced by hot compression of $50 \%$ or $75 \%$ at a strain rate of $1 / \mathrm{s}$ in a single pass at $1023 \mathrm{~K}$, the microstructural evolution behaviors in the positions with and without the shear strain were compared.

As the equivalent plastic strain increased, the fraction of high angle grain boundaries with misorientations between $15^{\circ}$ and $30^{\circ}$ increased in the similar way regardless of the presence of shear strain. On the other hand, the fraction of boundaries with misorienations in excess of $30^{\circ}$ increased mainly at the expense of LAGB. Such the expense occurred at much higher rate with shear strain than without shear strain.

Under the same equivalent strain of 1.5 , the principal strains and the rotation angle $\phi$ from the $x$ and $y$ axes to the principal axes at each site were calculated. Though the deformed austenite grains had a similar thickness, the rotation angle of compressive direction became larger with increasing shear strain. Because of the rotation of compressive direction during deformation, a kind of multidirectional deformation seemed to occur with an increase in shear strain. Consequently, the multidirectional deformation accelerated the subdivision of austenite grain interiors with increased misorientations between subdivided local areas.

\section{Acknowledgements}

The authors would like to thank Ms. Sakurai, NIMS, for her help with EBSD experiment. One of the authors (J.Y.C) was supported by the Post-doctoral Fellowship Program of KOREA SCIENCE \& Engineering Foundation (KOSEF) and wishes to express sincere gratitude to the Steel Research Center, NIMS.

\section{REFERENCES}

1) V. M. Segal: Mater. Sci. Eng. A 197 (1995) 157-164.

2) Y. Iwahashi, Z. Horita, M. Nemoto and T. G. Langdon: Acta Mater. 45 (1997) 4733-4741.

3) D. H. Shin, B. C. Kim, Y.-S. Kim and K.-T. Park: Acta Mater. 48 (2000) 2247-2255.

4) A. Belyakov, T. Sakai, H. Miura and R. Kaibyshev: ISIJ Int. 39 (1999) 
592-599.

5) Y. Saito, N. Tsuji, H. Utsunomiya, T. Sakai and R. G. Hong: Scr. Mater. 39 (1998) 1221-1227.

6) Y. Saito, N. Tsuji, H. Utsunomiya and T. Sakai: Acta Mater. 47 (1999) 579-583.

7) N. Tsuji, Y. Saito, H. Utsunomiya, S. Tanigawa: Scr. Mater. 40 (1999) 795-800.

8) Z. Horita, D. Smith, M. Furukawa, M. Nemoto, R. Z. Valiev and T. G. Langdon: J. Mater. Res. 11 (1996) 1880-1890.

9) J. G. Sevillano, P. Van Houtte and E. Aernoudt: Prog. Mater. Sci. 25 (1980) 69-134.

10) T. Inoue, S. Torizuka, K. Nagai, K. Tsuzaki and T. Ohashi: Mater. Sci. Tech. 17 (2001) 1580-1588

11) T. Inoue, S. Torizuka and K. Nagai: Mater. Sci. Tech. 18 (2002) 1007 1015.

12) P. J. Hurley, B. C. Muddle and P. D. Hodgson: Metall. Mater. Trans.
32A (2001) 1507-1517.

13) ABAQUS/Explicit ver. 5.8, User's manual, (Hibbit, Karlsson \& Sorensen, Inc. USA, 1998).

14) J. R. Bowen, P. B. Prangnell and F. J. Humphreys: Mater. Sci. Tech. 16 (2000) 1246-1250.

15) D. A. Hughes and N. Hansen: Acta Mater. 45 (1997) 3871-3886.

16) P. B. Prangnell, J. R. Bowen, A. Gholinia and M. V. Markushev: International Symposium on Superplasticity, ed. by P. B. Berbon et al., (MRS Fall Meeting, Boston, 1999) 323-334.

17) A. Gholinia, P. B. Prangnell and M. V. Markushev: Acta Mater. 48 (2000) 1115-1130.

18) A. Belyakov, T. Sakai, H. Miura and K. Tsuzaki: Philos. Mag. A 81 (2001) 2629-2643.

19) Z. C. Wagn and P. B. Prangnell: Mater. Sci. Eng. A328 (2002) 87-97.

20) P. J. Apps, J. R. Bowen and P. B. Prangnell: Acta Mater. 51 (2003) 2811-2822. 